\title{
Hämolytische Anämie bei nicht identischer Blutgruppe
}

Quelle: Bakr MA, Abbas TM, Mustafa A, Ghoneim MA. Hemolytic anemia after $\mathrm{ABO}$ nonidentical living donor kidney transplantation.Clin Exp Nephrol 2009; Jan 20 [in press]

Thema: AB0-kompatible Nierentransplantate mit nicht identischer Blutgruppe werden häufig genutzt. Eine Folge nach Transplantationen mit nicht identischen Blutgruppen kann die erworbene hämolytische Anämie sein. Patienten mit den Blutgruppen $A, B$ oder $A B$ können Organe von AB0-kompatiblen und bezüglich der Blutgruppe nicht identischen Spendern erhalten, vor allem wenn diese die Blutgruppe 0 haben. Auch AB-positive Patienten, die Nieren von Spendern mit den Blutgruppen A oder B bekommen, können eine hämolytische Anämie entwickeln.

Projekt: Insgesamt wurde in 214 Fällen eine Lebendnierentransplantation bei nicht identischer Blutgruppe durchgeführt. Alle in die Studie eingeschlossenen Patienten bekamen Nieren von Organspendern mit einem HLA-Mismatch (HLA: „Human Leukozyte Antigen“) und erhielten vor der Transplantation unspezifische Bluttransfusionen. Unter den Patienten waren 164 Männer und 50 Frauen. Zehn Patienten mit einer CyA-basierten Therapie (CyA: Cyclosporin A) entwickelten eine Hämolyse. Die CyAGabe wurde bei Patienten mit 3-facher Immunsuppression (Prednisolon, CyA, Azathioprin) abgebrochen und bei Patienten unter einer Prednisolon-/CyA-Therapie zu einer Azathioprinbehandlung umgewandelt. Bei allen Patienten waren vor der Transplantation der Antikörperscreen, der direkte Antiglobulintest und zytotoxische Cross-matchReaktionen negativ.

Ergebnis: Bei 9 Patienten war die Prognose sehr gut, einer starb an einer schweren Hämolyse. Am häufigsten trat die Hämolyse bei Empfängern mit der Blutgruppe A auf (60\%) und die schwersten Fälle ereigneten sich in der B-positiven Patientengruppe. Sechs Patienten erhielten Antigen negative rote Blutzellen. Wie eine multivariable Analyse zeigte, haben die primäre Immunsuppression, die Zeit bis zum Beginn der Diurese und die Donorblutgruppe einen signifikanten Einfluss auf die Entwicklung einer Hämolyse.

Fazit: Die Hämolyse nach einer Nierentransplantation kommt nicht sehr häufig vor. Sie kann jedoch auftreten, wenn der Spender AB0-kompatibel ist und seine Blutgruppe nicht mit der des Empfängers übereinstimmt. Die Blutgruppe des Nierendonors und -spenders, die Zeit bis zum Beginn der Diurese und die primäre Immunsuppression (vor allem CyA) sind signifikante Risikofaktoren für die Entwicklung einer Anämie. Die Auswirkungen der Hämolyse auf den Patienten sind gewöhnlich nicht sehr stark und die Erkrankung kann mit einer Änderung der Immunsuppression (kein CyA mehr geben) behandelt werden.

Key words: Lebendnierentransplantation - nicht identische Blutgruppen - ABO - Anämie - Hämolyse 